\title{
Treadmill exercise alleviates diabetic cardiomyopathy by suppressing plasminogen activator inhibitor expression and enhancing eNOS in streptozotocin-induced male diabetic rats
}

\author{
Wang Chengji and Fan Xianjin \\ College of Physical Education, Chaohu University, Anhui Province, China \\ Correspondence should be addressed to F Xianjin: shenxiazhao@126.com
}

\section{Abstract}

Objective: To investigate the biological mechanism of the effect of different intensity exercises on diabetic cardiomyopathy.

Methods: 87 raise specific pathogen SPF healthy 6-week-old male Sprague-Dawley rats, fed 6 weeks with high-fat diet for rats were used, and a diabetic model was established by intraperitoneal injection of streptozotocin - randomly selected 43 rats were divided into Diabetic control group (DCG, $n=10)$, Diabetic exercise group 1 (DEG1, $n=11$ ), Diabetic exercise group 2 (DEG2, $n=11$ ) and Diabetic exercise group 3 (DEG3, $n=11)$. The rats in DEG1 were forced to run on a motorized treadmill, the exercise load consisted of running at a speed of $10 \mathrm{~m} / \mathrm{min}$, the exercise load of the rats in DEG2 were running at a speed of $15 \mathrm{~m} / \mathrm{min}$, the exercise load of the rats in DEG3 were running at a speed of $20 \mathrm{~m} / \mathrm{min}$, for one hour once a day for 6 weeks. After 6 weeks of exercise intervention, glucose metabolism-related indexes in rats such as blood glucose (FBG), glycosylated serum protein (GSP) and insulin (FINS); cardiac fibrinolytic system parameters such as PAI-1 (plasminogen activator inhibitor 1), Von Willebrand factor (vWF), protein kinase C (PKC) and diacylglycerol (DAG); and serum level of NO, eNOS and T-NOS were measured. Result: Compared with DCG, fasting blood glucose and GSP were decreased, while insulin sensitivity index and insulin level were increased in all rats of the three exercise groups. FBG decrease was statistically significant $(P<0.01)$, only GSP decrease was statistically significant $(P<0.05)$ in DEG1 and DEG2, PAI-1 in three exercise groups were significantly reduced $(P<0.05)$, plasma vWF levels in the three exercise groups were significantly lower than those in the DCG group $(P<0.01)$; PKC levels decreased dramatically in the three exercise groups and DAG levels decrease slightly $(P<0.05)$, but with no significant difference. Compared with DCG, the serum level of NO was significantly higher $(P<0.05)$, and eNOS level was significantly elevated $(P<0.05)$. T-NOS elevation was statistically significant in DEG1 $(P<0.05)$. Conclusions: Low- and moderate-intensity exercise can better control blood glucose level in diabetic rats; myocardial PAI-1 in DEG1, DEG2 and DEG3 rats decreased significantly $(P<0.05)$, serum NO increased $(P<0.05)$ and eNOS increased $(P<0.05)$ significantly. Therefore, it is inferred that exercise improves the biological mechanism of diabetic cardiomyopathy by affecting the levels of PAI- 1 and eNOS, and there is a dependence on intensity. 


\section{Introduction}

Smoking (1), low physical activity (2), obesity, insulin resistance and hyperglycemia (3) are independently associated with incident type 2 diabetes mellitus (T2DM). Insulin resistance is affected by metabolic risk factors for both T2DM and cardiovascular disease, including overall obesity, central obesity, elevated triglyceride levels, low HDL levels, hyperglycemia and hypertension (4). Hyperglycemia has been shown to stimulate coagulation in healthy humans, and hyperinsulinemia has been associated with impaired fibrinolysis (5). Diabetic patients have elevated levels of coagulation factors and impaired fibrinolysis, inducing a hypercoagulable state that may contribute to the increased risk of atherothrombotic events and venous thromboembolism $(6,7,8,9)$. Although reducing fasting plasma glucose levels to normal is seen as a way to prevent negative cardiovascular outcomes, such as myocardial infarction or stroke (10), the most recent study with insulin-treated T2DM patients failed to prove any protective effect of tight glycemic control (11).

High levels of tPA have been shown to be predictive of future T2DM independent of metabolic syndrome (12, 13). Another study showed that tPA and PAI-1 levels are similar in patients with newly diagnosed T2DM compared to patients with T2DM for a long duration (14). These findings indicate that impaired fibrinolysis in diabetic patients precedes the manifest diagnosis.

Some investigations have demonstrated that PAI-1 is predictive of T2DM but that its predictive ability disappears after adjusting for markers of metabolic syndrome $(15,16)$. This observation suggests that high plasma PAI-1 levels are associated with factors involved in metabolic syndrome, mainly obesity (17). Other studies have indicated that high baseline PAI-1 levels are associated with incident diabetes (18) and that PAI-1 levels continue to increase with increasing glucose levels and the development of T2DM $(19,20)$. The tPA/PAI-1 complex, tPA bound to PAI-1, has been associated with cardiovascular disease $(21,22)$, but its relation to incident diabetes is unknown.

Elevated von Willebrand Factor (VWF) levels increase the risk of cardiovascular events in patients with T2DM (23) but have not been shown to be associated with incident T2DM (13). VWF is produced by endothelial cells, which may be activated by proinflammatory cytokines (24) such as IL-6, which in turn have been associated with an increased risk of incident T2DM (25). $\mathrm{C}$-reactive protein (CRP) has also been associated with an increased risk of incident T2DM, though this association was lost after adjusting for IL-6 (13). The hyperglycemiainduced activation of the diacylglycerol (DAG)-protein kinase C (PKC) pathway has multiple adverse effects on the vascular function. Hyperglycemia increases the levels of DAG, which in turn activates PKC. In hyperglycemic circumstances, DAG is synthesized from the glycolytic intermediates dihydroxyacetone phosphate (DHAP) and glycerylaldehyde-3-phosphate, by a de novo pathway (26). Oxidants like $\mathrm{H}_{2} \mathrm{O}_{2}$ can also activate the DAG/PKC pathway.

At present, exercise therapy is a safe, simple and effective method for the treatment of diabetes, and its effectiveness has been confirmed in basic research and clinical applications $(27,28)$. However, the report of cardiovascular fibrinolytic system is limited to the effect of exercise on blood PAI-1. In this study, diabetic rat model was established by a high-fat and sugar diet and intraperitoneal injection of streptozotocin (STZ), to observe the changes of PAI-1, vWF, PKC, DAG and serum NO, endothelial nitric oxide synthase (eNOS) and total nitric oxide synthase (T-NOS) in the myocardium of diabetic rats after different intensity exercises, to explore the effects of exercise on cardiovascular diastolic function and blood coagulation function in diabetic rats and to explore the biological mechanism of exercise for the prevention and treatment of diabetic cardiomyopathy.

\section{Materials and methods}

\section{Animal models and groups}

87 male SPF healthy 6-week-old male Sprague-Dawley rats, weighing $190 \pm 15 \mathrm{~g}$, were obtained from Shanghai Lab Animal Research Center. Free diet, feeding and exercise at room temperature $20 \sim 25^{\circ} \mathrm{C}$ and humidity $60 \sim 40 \%$, natural circadian rhythm, light enough.

After one-week adjustment period given high-fat diet for rats, its feed formula is lard 10\%, white sugar 20\%, egg yolk powder $5 \%$, bile salt $0.2 \%$, vitamin $0.05 \%$, mineral $0.2 \%$ and basic feed $64.55 \%$. According to previous studies (29), rats were fed with high-fat diet and established diabetic rat model. That is, after 6 weeks of feeding, all high-fat diet rats were fasted for $12 \mathrm{~h}$ after measuring the weight and were made diabetic by a single injection of STZ $(30 \mathrm{mg} / \mathrm{kg}$, i.v.) dissolved in citrate buffer $(20 \mathrm{mg} / \mathrm{dL})$, $\mathrm{pH}$ 4.5. The prepared STZ solution was injected within $30 \mathrm{~min}$. 
Rats were considered diabetic when blood glucose levels were $>300 \mathrm{mg} / \mathrm{dL}$ five days later. Eighty-one rats were established as model successfully. Forty-three were randomly selected from the successful model of diabetic rats and were divided into 4 groups: Diabetic control group (DCG, $n=10$ ), Diabetes mellitus + exercise group 1 (DEG1, $n=11$ ), Diabetes mellitus + exercise group 2 (DEG2, $n=11$ ) and Diabetes mellitus + exercise group 3 (DEG3, $n=11$ ). The rats in the exercise groups were subjected to run on a treadmill, and according to the recommendation of Bedford et al. (11) were divided into lower, medium and great intensity, that is the DEG1 running at a speed of $10 \mathrm{~m} / \mathrm{min}$ (equivalent to $30 \% \mathrm{VO}_{2} \mathrm{max}$ ), DEG2 was $15 \mathrm{~m} / \mathrm{min}$ (equivalent to $50 \% \mathrm{VO}_{2} \max$ ) and $\mathrm{DEG} 3$ was $20 \mathrm{~m} / \mathrm{min}$ (equivalent to $70 \% \mathrm{VO}_{2} \max$ ), one hour once a day, 5 times a week for 6 weeks. High blood sugar, exercise and other reasons (such as renal necrosis) caused a small amount of death in the experimental rats, finally, the sample size of DCE, DEG1 and DEG3 were 10, respectively, DEG2 were 9. All experimental protocols were approved by the Animal Care and Use Committee of Chaohu University. The procedures were performed according to the recommendations of the Institutional Animal Care Committee.

\section{Sample collection and processing}

$24 \mathrm{~h}$ after the last exercise session and 12-h fasting, the rats were weighed. After taking the blood from the tail vein, the rats were anesthetized with $10 \%$ chloral hydrate, and then blood was collected through abdominal aorta, and maintaining static $1 \mathrm{~h}$ at room temperature, samples were centrifuged at $1590 \mathrm{~g}$ for $20 \mathrm{~min}$, stored at $-70^{\circ} \mathrm{C}$ and directly analyzed. $0.5 \mathrm{~g}$ of myocardial tissue was taken, washed with ice-cold saline and after draining water by filter paper and weighing, physiological saline of 1:9 ratio was added. Then, homogenized for $10 \mathrm{~s} \times 3$ times on homogenizer at $10,000 \mathrm{rpm}(8300 \boldsymbol{g})$ to prepare $10 \%$ homogenate, and tissue homogenates were centrifuged at $671 \mathrm{~g}$ for $10 \mathrm{~min}$ at $4^{\circ} \mathrm{C}$ and the supernatants were obtained for testing the level of PAI-1 and vWF in cardiac muscle.

\section{Index testing}

Blood glucose (FBG) was tested using the Japanese Kyoto GT-1640 blood glucose meter, and glycated hemoglobin was assessed using glycated hemoglobin (A1C) test kit in accordance with the manufacturer's instructions. ELISA was used to test serum insulin (FINS), serum NO, eNOS, T-NOS, PAI-1, vWF PKC and DAG in cardiac muscle tissue. The insulin resistance index (IRI) was calculated using the international general formula of HOMA (12):

$$
\mathrm{IRI}=(\mathrm{FBG} \times \mathrm{FINS}) \div 22.5
$$

\section{Statistical analysis}

All values were expressed as means \pm s.E.M., and the statistical analysis was carried out using statistical package (version 20.0 for Windows; SPSS). To compare the differences between the groups mean, one-way analysis of variance was performed. The statistical significance level was set at $P<0.05$, and the very significant difference was set at $P<0.01$.

\section{Results}

The comparison of FBG, GSP, FINS and IRI in rats of each group is shown in Table 1. After 6 weeks of experiment, compared with DCG group, FBG in the three exercise groups decreased significantly $(P<0.05)$, GSP in each exercise group decreased by varying degrees, among them, the GSP in DEG1 and the DEG 2 groups decreased significantly $(P<0.05)$, FINS in exercise group was increased, but only the increase in DEG3 group was statistically significant $(P<0.05)$, and the FINS in DEG1 and DEG2 groups was significantly lower than that in the DEG3 group $(P<0.05)$;

Table 1 Comparison of fasting blood glucose, glycosylated serum protein, insulin and insulin resistance index in each group.

\begin{tabular}{lr}
\hline Groups & $\boldsymbol{n}$ \\
\cline { 1 - 1 } DCG & 10 \\
LIG & 10 \\
MIG & 9 \\
HIG & 10 \\
\hline
\end{tabular}

\begin{tabular}{l} 
FBG $(\mathrm{mmol} / \mathrm{L})$ \\
\hline $16.66(4.8)$ \\
$10.17(5.7)^{* *}$ \\
$10.78(0.8)^{* *}$ \\
$12.03(4.4)^{* *}$ \\
\hline
\end{tabular}

\begin{tabular}{c} 
GSP $(\mathrm{mmol} / \mathrm{L})$ \\
\hline $3.62(0.49)$ \\
$2.99(1.0)^{*}$ \\
$2.90(1.5)^{*}$ \\
$3.21(0.4)$ \\
\hline
\end{tabular}

\begin{tabular}{c}
\hline FINS $(\mathrm{mmol} / \mathrm{L})$ \\
\hline $3.42(0.5)$ \\
$3.94(0.4)^{\#}$ \\
$3.73(0.8)^{\#}$ \\
$4.58(1.3)^{*}$
\end{tabular}

\begin{tabular}{l}
\multicolumn{1}{c}{ IRI } \\
\hline $2.48(0.6)$ \\
$1.77(1.0)^{*}, \# \#$ \\
$1.79(0.4)^{*}, \#$ \\
$2.51(1.2)$
\end{tabular}

Compared with the DCG group, ${ }^{*} P<0.05, * * P<0.01$; compared with the DEG 3 group, ${ }^{\#} P<0.05,{ }^{\# \#} P<0.01$.

(c) 2018 The authors Published by Bioscientifica Ltd

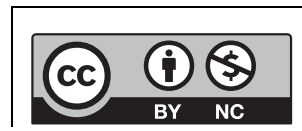

This work is licensed under a Creative Commons Attribution-NonCommercial 4.0 International License. 
IRI of DEG1 and DEG2 groups significantly decreased $(P<0.05)$, but that of DEG3 group increased, and there was no significant difference compared with the DCG group $(P>0.05)$, and significantly higher than that of DME1 and DME2 groups $(P<0.01, P<0.05)$.

The comparison of NO, eNOS and T-NOs in rats of each group are shown in Table 2. After 6 weeks of experiment, compared with DCG group, the serum levels of NO were significantly increased in all exercise groups $(P<0.05, P<0.01)$, the serum levels of eNOS were significantly higher $(P<0.05, P<0.01)$ and T-NOS of DEG1 group was significantly higher $(P<0.05)$.

The comparison of vWF, PAI-1, PKC and DAG in rats of each group are shown in Table 3. Compared with the DCG group, the plasma vWF level of the three exercise groups of rats was significantly reduced $(P<0.05, P<0.01)$, compared with the DCG group, the plasma PAI-1 level reduced significantly $(P<0.05, P<0.01)$ in three exercise groups rats, also the PKC and DAG levels reduced significantly $(P<0.05, P<0.01)$.

\section{Discussion}

The effect of exercise intensity on FBG, GSP, FINS and IRI in diabetic rats

It is always been known that control blood glucose levels is the key to treat diabetes and prevent diabetic complications. The results show that after 6 weeks of different-intensity treadmill exercise for diabetic rats, fasting blood glucose and GSP were decreased by varying degrees; however, the effect of low- and moderateintensity exercise was significant. In this regard, low- and moderate-intensity exercise is more suitable to control blood glucose in patients with diabetes. Also insulin levels in DEG1 and DEG2 groups had a tendency to increase, but with no statistical significance; however, IRI declined significantly, the results showed that the 6-week lowand moderate-intensity treadmill exercise could improve insulin sensitivity and reduce the degree of insulin resistance in rats; its effect is related to the intensity of

Table 2 Comparison of NO, eNOS, T-NOS in each group.

\begin{tabular}{|c|c|c|c|c|}
\hline Groups & $N$ & NO $(\mu \mathrm{mol} / \mathrm{L})$ & eNOS $(\mu \mathrm{mol} / \mathrm{L})$ & T-NOS $(\mu \mathrm{mol} / \mathrm{L})$ \\
\hline DCG & 10 & $4.22(1.7)$ & $9.87(3.5)$ & $25.17(5.1)$ \\
\hline LIG & 10 & $6.78(2.1)^{*}$ & $14.67(3.8) *$ & $32.6(4.1)^{*}$ \\
\hline MIG & 9 & $8.96(2.2)^{* *}$ & $22.16(5.8) * *$ & 38.07 (13.9) \\
\hline HIG & 10 & $7.39(3.8)$ * & $23.75(11.1) * *$ & 37.74 (16.6) \\
\hline
\end{tabular}

Compared with the DCG group, $* P<0.05, * * P<0.01$.

$$
\text { http://www.endocrineconnections.org }
$$

exercise. The potential mechanism of aerobic exercise is a kind of high energy consumption, and can enhance the cellular uptake and utilization of glucose and improve the insulin sensitivity of the body tissue cells.

\section{Effect of different intensity exercises on PAI-1 and vWF content in myocardium of diabetic rats}

Plasminogen activator inhibitor-1 (PAI-1) is the primary physiological inhibitor of endogenous fibrinolysis that acts via inhibition of the tissue plasminogen activator (tPA) and the urokinase type activator (uPA), often leading to fibrin accumulation in basement membranes and interstitial tissues (30). Elevations in plasma PAI-1 appear to compromise normal fibrin clearance mechanisms and promote thrombosis. In large epidemiological studies, elevated plasma PAI-1 has been demonstrated in various subgroups as an important feature of T2D and MetS (19).

Some studies have found that acute exercise rapidly increases plasma fibrinolytic activity, and its effect is related to the intensity and duration of exercise. Submaximal exercise can make moderate elevation of fibrinolytic activity. In addition, the effect of different exercise intensities on PAI-1 was different - low-intensity exercise has little effect on the activity of PAI-1, exhaustive exercise can increase PAI-1 activity, while moderate exercise can reduce the activity of PAI-1, and effectively prevent thrombotic diseases $(31,32)$. Our results demonstrated that after 6 weeks of exercise training, compared with the control group, the myocardial PAI-1 in each exercise group decreased significantly. This is consistent with abovementioned findings. However, because of the lack of normal control group, the biological mechanism of reducing myocardial PAI-1, improving fibrinolytic system and preventing diabetic cardiomyopathy by different intensity exercises remains to be discussed.

Von Willebrand factor (vWF) is a glycoprotein synthesized and secreted by vascular endothelial cells and stored in Weibel-Palade bodies, involved in coagulation, hemostasis, platelet aggregation and adhesion. When the vascular endothelial cells are normal, the plasma vWF content is small, and when the vascular endothelial cells are damaged, the release into the blood is increased and is now recognized as a marker of endothelial cell damage and dysfunction, is the most valuable regulatory factors produced by endothelial cells. It has been shown that diabetic patients are hypercoagulable and their vWF levels are significantly higher than those of non-diabetic healthy adults, indicating that endothelial dysfunction exists in diabetic patients. At present, there are few studies on the

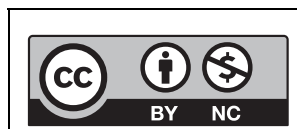

This work is licensed under a Creative Commons Attribution-NonCommercial 4.0 International License. 
Table 3 Comparison of PAI-1, PKC, DAG and VWF in each group.

\begin{tabular}{|c|c|c|c|c|c|}
\hline Groups & $n$ & PAI-1 $(\mu \mathrm{mol} / \mathrm{L})$ & PKC (ng/mg) & DAG (ng/mg) & vWF $(n g / m L)$ \\
\hline DCG & 10 & $7.71(0.5)$ & $0.38(0.1)$ & $0.72(0.2)$ & $15.03(1.9)$ \\
\hline LIG & 10 & $7.05(0.6)$ * & $0.27(0.1)$ * & $0.53(0.2)$ * & $11.96(2.4) *$ \\
\hline MIG & 9 & $6.48(0.5) * *$ & $0.24(0.1)$ * & $0.53(0.1)$ * & $9.93(1.9)$ * \\
\hline HIG & 10 & $6.24(0.6)^{* *}$ & $0.20(0.1)^{*}$ & $0.46(0.2)$ * & $9.17(2.5)$ * \\
\hline
\end{tabular}

Compared with the DCG group, $* P<0.05, * * P<0.01$.

effect of exercise training on plasma vWF concentration. Studies by Hilberg et al. (33) showed a significant decrease in plasma vWF concentrations in 24 women after 12 weeks of aerobic exercise. At the same time, some studies have found that training can reduce the expression of vWF on the body platelets and thus reduce the platelet aggregation caused by the change of shear force in the body during quiet and large-load acute exercise (34). Our results demonstrated that after 6 weeks of exercise training, compared with the control group, the myocardial vWF in each exercise group decreased significantly. It shows that exercise can relieve the injury degree of endothelial cells, improve the function of fibrinolytic system, protect endothelial function and prevent diabetic complications.

\section{Effect of different intensity exercises on the DAG/ PKC pathway in myocardium of diabetic rats}

Diabetes mellitus is a complex syndrome of multiple disorders including vascular dysfunction. PKC could play a role in diabetes-related vascular pathology through multiple mechanisms including cell growth and proliferation, cell permeability, oxidative stress, increased vascular reactivity, inhibition of $\mathrm{K}^{+}$channels and $\mathrm{Na}^{+}$$\mathrm{K}^{+}$-ATPase, activation of cytosolic phospholipase A2, vascular remodeling and increased ECM and vascular inflammation and increased proinflammatory cytokines (35). In diabetes, PKC is activated by advanced glycation end (AGE) products and polyol pathway flux (36). Also, chronic hyperglycemia stimulates synthesis of DAG and activates DAG-dependent cPKCs and nPKCs in cultured bovine aortic endothelial cells and VSM (37). Fatty acids, especially the unesterified forms and their coenzyme A (CoA) esters, work synergistically with DAG to activate PKC (38). Exercise improves glycemic control in patients with diabetes, thereby alleviating glycolysis caused by hyperglycemia and reducing the DAG content. Our results demonstrated that after 6-week exercise training, compared with the control group, the myocardial PKC and DAG levels in each exercise group decreased significantly. The results showed that exercise decreased

http://www.endocrineconnections.org https://doi.org/10.1530/EC-18-0060

(c) 2018 The authors Published by Bioscientifica Ltd blood glucose level and DAG synthesis in type 2 diabetic rats, thereby reducing the activity of $\mathrm{PKC}$, to reduce the degree of vascular endothelial injury, and endothelial cells have a protective effect, suggesting. This may be one of the mechanisms for the prevention and treatment of diabetes and its complications.

Effects of different intensity exercises on serum NO, eNOS and T-NOS in diabetic rats

To a certain extent, the level of NO reflects the degree of injury in diabetic cardiomyopathy, and its metabolic disorders may be one of the mechanisms leading to diabetic cardiomyopathy (39). NOs is a key enzyme in the synthesis of NO, there are 3 subtypes: neuronal nitric oxide synthase (nNOS), inducible nitric oxide synthase (iNOS) and endothelial nitric oxide synthase (eNOS). Catalytic production of NO by iNOS plays a strong free radical effect; however, eNOS catalyzes the production of $\mathrm{NO}$ as a potent endogenous vascular relaxing factor, has a strong protective effect on blood vessels, inhibits smooth muscle cell proliferation, reduces platelet adhesion, inhibits lipid peroxidation and production of oxygen free radicals, to protect cells from the damage of superoxide anion (39).

Long-term aerobic exercise not only increases the activity of eNOS, but also increases the expression of mRNA eNOS, increase the amount of NO synthesis, promote the endothelium-dependent relaxation response and then prevent cardiovascular disease (40). The study by Archana found that 12 weeks of the aerobic exercise can improve the heart rate variability in patients with diabetes, and at the same time, increased plasma NO levels (41). The results of this study show that after 6 weeks of exercise in diabetic rats, the eNOS of each exercise group was significantly higher than that of DCG group. Only T-NOS of DEG1 significantly increased, and this may be related to the fact that T-NOS is the sum of the three kinds of NOS, the influence factors are more, with large difference in individual factors. T-NOS not being high also indirectly indicates that lower iNOS level is advantageous 
for cardiac muscle tissue. In addition, the serum levels of NO were significantly increased in the 3 intensity exercise groups; this study was consistent with the results of the study by Archana (41), and the changes of eNOS and $\mathrm{NO}$ are dependent on the intensity; moderate-intensity exercise is more beneficial to improve the level of serum NO in diabetic rats.

\section{Conclusions}

6 weeks of different intensity aerobic exercises can reduce the PAI-1 content of myocardium in diabetic rats, increase serum eNOS activity and NO level, and therefore, can prevent and improve diabetic cardiomyopathy. The effect of 6 weeks of different intensity aerobic exercises on myocardial PAI-1 and serum eNOS activity and NO level in diabetic rats was dependent on the intensity.

\section{Declaration of interest}

The authors declare that there is no conflict of interest that could be perceived as prejudicing the impartiality of the research reported.

\section{Funding}

This work was supported by college of Physical Education.

\section{Author contribution statement}

Wang Chengji participated in the study design, analysis, report development and interpretation of study findings. Fan Xianjin participated in writing the report.

\section{References}

1 Willi C, Bodenmann P, Ghali WA, Faris PD \& Cornuz J. Active smoking and the risk of type 2 diabetes: a systematic review and meta-analysis. JAMA 2007298 2654-2664. (https://doi.org/10.1001/ jama.298.22.2654)

2 Hu FB, Li TY, Colditz GA, Willett WC \& Manson JE. Television watching and other sedentary behaviors in relation to risk of obesity and type 2 diabetes mellitus in women. JAMA 2003289 1785-1791. (https://doi.org/10.1001/jama.289.14.1785)

3 Norberg M, Stenlund H, Lindahl B, Andersson C, Weinehall L, Hallmans G \& Eriksson JW. Components of metabolic syndrome predicting diabetes: no role of inflammation or dyslipidemia. Obesity 200715 1875-1885. (https://doi.org/10.1038/oby.2007.222)

4 Meigs JB. Epidemiology of the insulin resistance syndrome. Current Diabetes Reports 20033 73-79. (https://doi.org/10.1007/s11892-0030057-2)

5 Stegenga ME, van der Crabben SN, Levi M, de Vos AF, Tanck MW, Sauerwein HP \& van der Poll T. Hyperglycemia stimulates coagulation, whereas hyperinsulinemia impairs fibrinolysis in healthy humans. Diabetes 200655 1807-1812. (https://doi. org/10.2337/db05-1543)

6 Lemkes BA, Hermanides J, Devries JH, Holleman F, Meijers JC \& Hoekstra JB. Hyperglycemia: a prothrombotic factor? Journal of Thrombosis and Haemostasis 20108 1663-1669. (https://doi. org/10.1111/j.1538-7836.2010.03910.x)

7 Carr ME. Diabetes mellitus: a hypercoagulable state. Journal of Diabetes and its Complications 200115 44-54. (https://doi. org/10.1016/S1056-8727(00)00132-X)

8 Petrauskiene V, Falk M, Waernbaum I, Norberg M \& Eriksson JW. The risk of venous thromboembolism is markedly elevated in patients with diabetes. Diabetologia 200548 1017-1021. (https://doi. org/10.1007/s00125-005-1715-5)

9 Jax TW, Peters AJ, Plehn G \& Schoebel FC. Hemostatic risk factors in patients with coronary artery disease and type 2 diabetes - a two year follow-up of 243 patients. Cardiovascular Diabetology 2009848. (https://doi.org/10.1186/1475-2840-8-48)

10 American Diabetes Association. Implications of the United Kingdom prospective diabetes study. Diabetes Care 200023 (Supplement 1) S27-S31. (https://doi.org/10.2337/diacare.26.2007.S28)

11 Gerstein HC, Bosch J, Dagenais GR, Díaz R, Jung H, Maggioni AP, Pogue J, Probstfield J, Ramachandran A, Riddle MC, et al. Basal insulin and cardiovascular and other outcomes in dysglycemia. New England Journal of Medicine 2012367 319-328. (https://doi. org/10.1056/NEJMoa1203858)

12 Eliasson MC, Jansson JH, Lindahl B \& Stegmayr B. High levels of tissue plasminogen activator (tPA) antigen precede the development of type 2 diabetes in a longitudinal population study. The Northern Sweden MONICA study. Cardiovascular Diabetology 2003219. (https://doi.org/10.1186/1475-2840-2-19)

13 Wannamethee SG, Sattar N, Rumley A, Whincup PH, Lennon L \& Lowe GD. Tissue plasminogen activator, von Willebrand factor, and risk of type 2 diabetes in older men. Diabetes Care 200831 995-1000. (https://doi.org/10.2337/dc07-1569)

14 Heldgaard PE, Henriksen JE, Sidelmann JJ, de Fine Olivarius N, Siersma VD \& Gram JB. Similar cardiovascular risk factor profile in screen-detected and known type 2 diabetic subjects. Scandinavian Journal of Primary Health Care 201129 85-91. (https://doi.org/10.310 9/02813432.2011.565164)

15 Anand SS, Yi Q, Gerstein H, Lonn E, Jacobs R, Vuksan V, Teo K, Davis B, Montague P, Yusuf S, et al. Relationship of metabolic syndrome and fibrinolytic dysfunction to cardiovascular disease. Circulation 2003108 420-425. (https://doi.org/10.1161/01. CIR.0000080884.27358.49)

16 Juhan-Vague I, Pyke SD, Alessi MC, Jespersen J, Haverkate F \& Thompson SG. Fibrinolytic factors and the risk of myocardial infarction or sudden death in patients with angina pectoris. ECAT study group. European concerted action on thrombosis and disabilities. Circulation 199694 2057-2063. (https://doi. org/10.1161/01.CIR.94.9.2057)

17 Eliasson M, Evrin PE \& Lundblad D. Fibrinogen and fibrinolytic variables in relation to anthropometry, lipids and blood pressure. The Northern Sweden MONICA Study. Journal of Clinical Epidemiology 199447 513-524. (https://doi.org/10.1016/0895-4356(94)90298-4)

18 Festa A, D'Agostino R Jr, Tracy RP \& Haffner SM. Elevated levels of acute-phase proteins and plasminogen activator inhibitor-1 predict the development of type 2 diabetes. Diabetes 200251 1131-1137. (https://doi.org/10.2337/diabetes.51.4.1131)

19 Festa A, Williams K, Tracy RP, Wagenknecht LE \& Haffner SM. Progression of plasminogen activator inhibitor-1 and fibrinogen levels in relation to incident type 2 diabetes. Circulation 2006113 1753-1759. (https://doi.org/10.1161/ CIRCULATIONAHA.106.616177)

20 Haffner SM. Insulin resistance, inflammation, and the prediabetic state. American Journal of Cardiology 200392 18J-26J. (https://doi. org/10.1016/S0002-9149(03)00612-X)

21 Johansson L, Jansson JH, Boman K, Nilsson TK, Stegmayr B \& Hallmans G. Tissue plasminogen activator, plasminogen activator inhibitor-1, and tissue plasminogen activator/plasminogen activator inhibitor-1 complex as risk factors for the development 
of a first stroke. Stroke 200031 26-32. (https://doi.org/10.1161/01. STR.31.1.26)

22 Wiman B, Andersson T, Hallqvist J, Reuterwall C, Ahlbom A \& deFaire U. Plasma levels of tissue plasminogen activator/ plasminogen activator inhibitor-1 complex and von Willebrand factor are significant risk markers for recurrent myocardial infarction in the Stockholm Heart Epidemiology Program (SHEEP) study. Arteriosclerosis, Thrombosis, and Vascular Biology 200020 2019-2023. (https://doi.org/10.1161/01.ATV.20.8.2019)

23 Frankel DS, Meigs JB, Massaro JM, Wilson PW, O'Donnell CJ, D'Agostino RB \& Tofler GH. Von Willebrand factor, type 2 diabetes mellitus, and risk of cardiovascular disease: the framingham offspring study. Circulation 2008118 2533-2539. (https://doi.org/10.1161/ CIRCULATIONAHA.108.792986)

24 Kerr R, Stirling D \& Ludlam CA. Interleukin 6 and haemostasis. British Journal of Haematology 2001115 3-12. (https://doi. org/10.1046/j.1365-2141.2001.03061.x)

25 Spranger J, Kroke A, Mohlig M, Bergmann MM, Ristow M, Boeing H $\&$ Pfeiffer AF. Adiponectin and protection against type 2 diabetes mellitus. Lancet 2003361 226-228. (https://doi.org/10.1016/S01406736(03)12255-6)

26 Way KJ, Katai N \& King GL. Protein kinase C and the development of diabetic vascular complications. Diabetic Medicine 200118 945-959. (https://doi.org/10.1046/j.0742-3071.2001.00638.x)

27 Aly YE, Abdou AS, Rashad MM \& Nassef MM. Effect of exercise on serum vitamin $\mathrm{D}$ and tissue vitamin $\mathrm{D}$ receptors in experimentally induced type 2 diabetes mellitus. Journal of Advanced Research 20167 671-679. (https://doi.org/10.1016/j.jare.2016.07.001)

28 Delevatti RS, Pinho CD, Kanitz AC, Alberton CL, Marson EC, Bregagnol LP, Lisboa SC, Schaan BD \& Kruel LF. Glycemic reductions following water- and land-based exercise in patients with type 2 diabetes mellitus. Complementary Therapies in Clinical Practice 201624 73-77. (https://doi.org/10.1016/j.ctcp.2016.05.008)

29 Meyer JD, Koltyn KF, Stegner AJ, Kim JS \& Cook DB. Influence of exercise intensity for improving depressed mood in depression: a dose-response study. Behavior Therapy 201647 527-537. (https://doi. org/10.1016/j.beth.2016.04.003)

30 Dellas C \& Loskutoff DJ. Historical analysis of PAI-1 from its discovery to its potential role in cell motility and disease. Thrombosis and Haemostasis 200593 631-640. (https://doi.org/10.1160/TH05-010033)

31 Sand KL, Flatebo T, Andersen MB \& Maghazachi AA. Effects of exercise on leukocytosis and blood hemostasis in 800 healthy young females and males. World Journal of Experimental Medicine 20133 11-20. (https://doi.org/10.5493/wjem.v3.i1.11)
32 Kahraman S, Bediz CS, Pişkin O, Aksu I, Topçu A, Yüksel F \& Demirkan F. The effect of the acute submaximal exercise on thrombin activatable fibrinolysis inhibitor levels in young sedentary males. Clinical and Applied Thrombosis/Hemostasis 201117 414-420. (https://doi.org/10.1177/1076029610385672)

33 Hilberg T, Nowacki PE, Muller-berghaus G \& Gabriel HH. Changes in blood coagulation and fibrinolysis associated with maximal exercise and physical conditioning in women taking low dose oral contraceptives. Journal of Science and Medicine in Sport 20003 383-390. (https://doi.org/10.1016/S1440-2440(00)80005-5)

34 Wang JS, Li YS, Chen JC \& Chen YW. Effects of exercise training and deconditioning on platelet aggregation induced by alternating shear stress in men. Arteriosclerosis, Thrombosis, and Vascular Biology 2005 25 454-446. (https://doi.org/10.1161/01.ATV.0000151987.04607.24)

35 Kim HR, Gallant C \& Morgan KG. Regulation of PKC autophosphorylation by calponin in contractile vascular smooth muscle tissue. BioMed Research International 20132013 358-643. (https://doi.org/10.1155/2013/310461)

36 Kizub IV, Klymenko KI \& Soloviev AI. Protein kinase C in enhanced vascular tone in diabetes mellitus. International Journal of Cardiology 2014174 230-242. (https://doi.org/10.1016/j.ijcard.2014.04.117)

37 Inoguchi T, Battan R, Handler E, Sportsman JR, Heath W \& King GL. Preferential elevation of protein kinase $C$ isoform beta II and diacylglycerol levels in the aorta and heart of diabetic rats: differential reversibility to glycemic control by islet cell transplantation. PNAS 199289 11059-11063. (https://doi. org/10.1073/pnas.89.22.11059)

38 Clarke M \& Dodson PM. PKC inhibition and diabetic microvascular complications. Best Practice and Research: Clinical Endocrinology and Metabolism 200721 573-586. (https://doi.org/10.1016/j. beem.2007.09.007)

39 Kleindienst A, Battault S, Belaidi E, Tanguy S, Rosselin M, Boulghobra D, Meyer G, Gayrard S, Walther G, Geny B, et al. Exercise does not activate the $\beta 3$ adrenergic receptor-eNOS pathway, but reduces inducible NOS expression to protect the heart of obese diabetic mice. Basic Research in Cardiology 2016111 40. (https://doi. org/10.1007/s00395-016-0559-0)

40 Gjovaag T, Hjelmeland AK, Oygard JB, Vikne H \& Mirtaheri P. Acute hemodynamic and cardiovascular responses following resistance exercise to voluntary exhaustion. Effects of different loadings and exercise durations. Journal of Sports Medicine and Physical Fitness 2016 56 616-623.

41 Archana R \& Mukilan R. Beneficial effect of preferential music on exercise induced changes in heart rate variability. Journal of Clinical and Diagnostic Research 201610 CC09-CC11.

Received in final form 12 February 2018

Accepted 19 March 2018

Accepted Preprint published online 19 March 2018 http://www.endocrineconnections.org https://doi.org/10.1530/EC-18-0060
(C) 2018 The authors Published by Bioscientifica Ltd

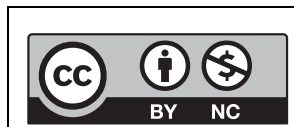

This work is licensed under a Creative Commons Attribution-NonCommercial 4.0 International License. 